\title{
Mechanising Blockchain Consensus
}

\author{
George Pîrlea \\ University College London, UK \\ george.pirlea.15@ucl.ac.uk
}

\author{
Ilya Sergey \\ University College London, UK \\ i.sergey@ucl.ac.uk
}

\begin{abstract}
We present the first formalisation of a blockchain-based distributed consensus protocol with a proof of its consistency mechanised in an interactive proof assistant.

Our development includes a reference mechanisation of the block forest data structure, necessary for implementing provably correct per-node protocol logic. We also define a model of a network, implementing the protocol in the form of a replicated state-transition system. The protocol's executions are modeled via a small-step operational semantics for asynchronous message passing, in which packages can be rearranged or duplicated.

In this work, we focus on the notion of global system safety, proving a form of eventual consistency. To do so, we provide a library of theorems about a pure functional implementation of block forests, define an inductive system invariant, and show that, in a quiescent system state, it implies a global agreement on the state of per-node transaction ledgers. Our development is parametric wrt. implementations of several security primitives, such as hash-functions, a notion of a proof object, a Validator Acceptance Function, and a Fork Choice Rule. We precisely characterise the assumptions, made about these components for proving the global system consensus, and discuss their adequacy. All results described in this paper are formalised in the Coq proof assistant.
\end{abstract}

\section{Introduction}

The notion of decentralised blockchain-based consensus is a tremendous success of the modern science of distributed computing, made possible by the use of basic cryptography, and enabling many applications, including but not limited to cryptocurrencies, smart contracts, application-specific arbitration, voting, etc.

In a nutshell, the idea of a distributed consensus protocol based on blockchains, or transaction ledgers, ${ }^{1}$ is rather simple. In all such protocols, a number of stateful nodes (participants) are communicating with each other in an asynchronous message-passing style. In a message, a node (a) can announce a transaction, which typically represents a certain event in the system, depending on

\footnotetext{
${ }^{1}$ Hereafter, we will be using the terms "(transaction) ledger" and "blockchain" interchangeably.
}

CPP 2018, January 08-09, 2018, Los Angeles, CA, USA

2018. ACM ISBN ...\$15.00

https://doi.org/ the previous state of the node or the entire network (we intentionally leave out the details of what can go into a transaction, as they are application-specific); a node can also (b) create and broadcast a block that contains the encoding of a certain vector of transactions, created locally or received via messages of type (a) from other nodes. Each recipient of a block message should then validate the block (i.e., check the consistency of the transaction sequence included in it), and, in some cases, append it to its local ledger, thus, extending its subjective view of the global sequence of transactions that have taken place in the system to date. The process continues as more messages are emitted and received.

In order to control the number of blocks in the system, distributed ledger protocols rely on certain cryptographic primitives, such as a hash-function hash defined both on transactions and blocks, a notion of a proof object necessary for defining the validity of a block, and an implementation of a Validator Acceptance Function (VAF) that is used to ensure that a block $b$ is valid wrt. to a proof object $p f$. Having a block $b$ and a proof object $p f$, one can check very fast whether $V A F b p f$ is true or false. What appears to be difficult is to produce an instance of a proof object $p f$, as it requires computing a pre-image of the hash function with respect to the current state of the local ledger of a specific node. The exact specifics of designing a $V A F$ and a discipline for minting blocks with $V A F$-valid proof objects, is a subject of active research, which is far beyond the scope of this paper, with the best known approaches being Proof-of-Work [8, 23] and Proof-of-Stake [2]. The computational hardness or probabilistic rarity of minting valid blocks is what controls the overall block population.

However, this setup by itself does not deliver a global consensus between the nodes. Indeed, in an asynchronous network, where messages can be rearranged, duplicated, or arbitrarily delayed, two different nodes $n_{1}$ and $n_{2}$ can receive different, or even conflicting, sets of valid blocks and decide to adopt them in their local ledgers. Assuming that initially all nodes share the same initial block (so-called Genesis Block), at any further state of the network, each two nodes' ledgers can be in a fork relation, when neither of them is a prefix of the other. The consensus is enabled by fixing a globally known Fork Choice Rule (FCR) function, that provides a decidable strict total order on all possible chains of blocks and is transitive and irreflexive. Thus, upon receiving a block, 
a node must check whether appending it to its local ledger is going to increase the ledger's "weight", and keep it if so, discarding it otherwise. Assuming every node follows the same FCR-imposed discipline for chain comparison, all participants will eventually share the same blockchain/transaction ledger instance.

Alas, the reality is a bit more complicated than the description above. For example, in a realistic fault-tolerant system implementation, nodes cannot afford to ignore blocks that arrive "out of order", which is not uncommon in an asynchronous setting. Not registering such blocks in a node's local state would pose serious liveness problems, as such nodes would be stuck with a "stale" local ledger, unable to progress along with the rest of the world. Furthermore, some nodes may not be active or known system-wide at the very beginning of communication, so they will start by first manifesting themselves, interacting only with a small set of peers they know. Finally, any node in the system should be able to request from its peers the set of publicly announced blocks these peers have witnessed in the past, so it would be possible for the node to "catch up" with the global state of the system, if, for instance, it has joined the network late or has been offline for some time.

In light of these and multiple other possible scenarios of distributed interaction, we believe that having a clean and principled model for rigorous formal reasoning about system-wide properties of distributed blockchain-based protocols is of paramount importance for gaining trust in the foundational principles of algorithms underlying, in particular, implementations of modern cryptocurrencies, such as Bitcoin [23], Ethereum [36] and Tezos [12].

In this work we provide such a model.

\subsection{Our Contributions}

The aim of this work is to develop a formal model of a blockchain-based consensus protocol, along with a set of necessary reference data structures and a network semantics, with an agenda to formally study its properties, abstracting away the implementation details of security-related primitives. Our contributions towards this goal include the following formal artifacts:

- A description of a minimal set of security primitives: hash, VAF, FCR, along with a set of laws (axioms) they should abide, and a discussion of these laws' adequacy wrt. real-world implementations;

- A reference implementation of block forests - a purely functional data structure implementing the local state of a node in the protocol in the presence of adopted out-of-order blocks, as well as a library of theorems about block forests, necessary for proving the consensus property of the system;
- A definition of a replicated state-transition machinery, implementing the per-node logic of the protocol, and semantics of the asynchronous network used for establishing protocol invariants;

- A formulated eventual consistency (global consensus) property for a blockchain network with a clique topology, a whole-system invariant implying the consensus in a quiescent state, and a proof of this invariant's inductivity, i.e., preservation by the network semantics.

In this work, we focus exclusively on system safety properties, i.e., proving that "nothing goes wrong". There are, indeed, more facts to establish about blockchain-based protocols, involving liveness ( $a k a$ chain growth), probabilistic irrevocability, stronger notions of consistency, and various security properties $[10,19]$. We do not address any of them in this paper, and consider statements and proofs of those properties as future applications of our formal model, discussing some of them in Section 7 .

Our Coq development is publicly available online [28]:

https://github.com/certichain/toychain

\subsection{Paper Outline}

In the remainder of the paper, we explain, by example, behaviours of blockchain-based networks and informally sketch intuition for system consistency (Section 2). We then describe the design and implementation of the core data structures, such as block forests, and their dependencies on the externally-provided security primitives in Section 3. In Section 4, we define the protocol machinery and the network semantics, elaborating on the statement and the proof of the consensus property in Section 5 . We report on our mechanisation experience and lessons learned in Section 6. We then discuss limitations and possible future applications of our model in Section 7 . We survey related verification and formalisation efforts in Section 8, and conclude in Section 9.

\section{Overview}

We begin by walking through an example that demonstrates interactions between nodes in a blockchain-based protocol and shows how consensus is achieved.

The goal of the consensus protocol is to guarantee that network participants agree wrt. the order in which transactions happened. This is achieved not by ordering transactions directly, but rather by grouping them into blocks and then agreeing, via $F C R$ - a comparison operation on block sequences (chains), which resulting blockchain to adopt. Assuming an agreement upon the rules of the protocol and initial state of the system, (i.e., everyone has the same Genesis Block $G B$ in the local state, as shown in Figure 1(a)), and provided everyone follows the rules, consensus is guaranteed once all block-carrying messages are delivered. 


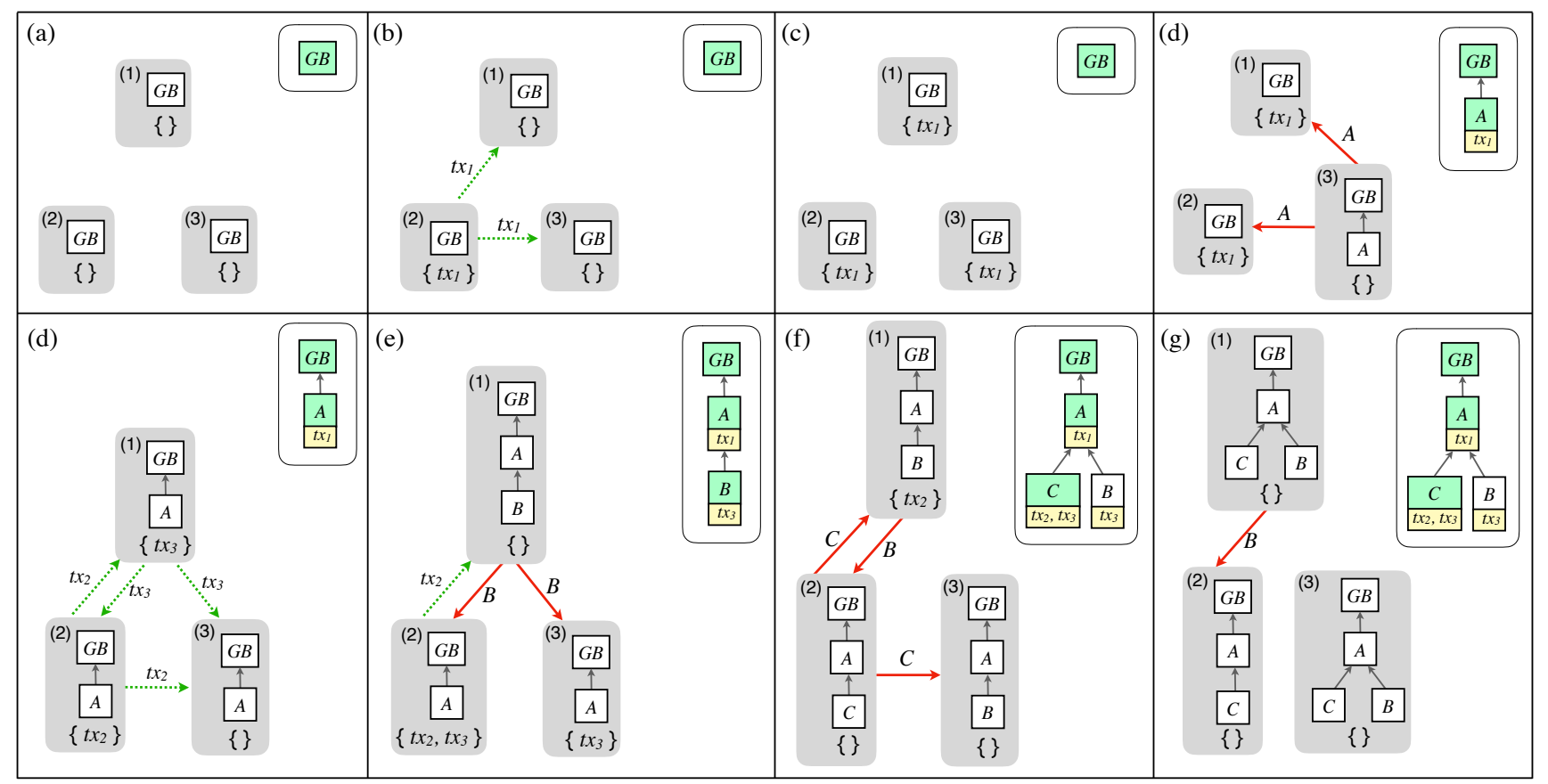

Figure 1. Progressive stages of interaction in a 3-node blockchain network, with "virtually shared" block-trees.

The stages (b)- $(\mathrm{g})$ of Figure 1 demonstrate further interactions in a 3-node blockchain-based system. Each stage also provides, in the top-right corner, the implicit "globally shared" tree of blocks, which will eventually be replicated in each of the participants and will serve to compute the globally shared ledger, thus delivering the desired ledger consensus. At any point in time, participants may issue transactions, which they broadcast to all their peers. For instance, a node (1) creates and announces a transaction $t x_{1}$ to its peers in (b). The messages containing the transactions propagate throughout the network, and each node temporarily stores them in its local transaction pool $\left(\left\{t x_{1}\right\}\right.$ in stage $\left.(\mathrm{c})\right)$. When "minting" (i.e., creating) a new block, nodes embed the transactions they know of inside the block and broadcast it to all participants in the network, as is done by node (3), which creates the block $A$ with the transaction $t x_{1}$ in (d), setting $A$ 's "parent block" to be $G B$.

Since the system is distributed, multiple transactions can be issued and propagated concurrently. As a result, when a block is minted, the minter does not necessarily know of all pending transactions, as the messages containing them might still be in transit. This is what happens in stage (e), where the node (1), which creates block $B$, has yet no knowledge of $t x_{2}$. This delay in propagation also might cause certain transactions to be included in different, "conflicting" blocks. For example, in (f) the transaction $t x_{3}$ has been included in both block $B$, minted previously by node (1), and block $C$, which is newly-minted by node (2). As such, looking at the global tree view in the top-right corner of (f), $t x_{3}$ is part of two blockchains: $[G B, A, C]$ and $[G B, A, B]$. However, this is not an issue for the global agreement: as FCR imposes a total order on blockchains, it uniquely determines which of the candidate chains is the correct one -in this case, $[G B, A, C]$ (we decide so for the sake of this example, indicating this by the green colour in the "shared" tree). Thus, all transactions, including $t x_{3}$, appear only once within the correct chain. As more messages propagate, more participants agree on $[G B, A, C]$ to be the "correct", i.e., canonical blockchain (g), until finally, everyone is agreement when the system is in a quiescent state with no messages in transit.

In the illustrative example above, we have seen how the system evolves over time and how it resolves conflicts by using the globally known $F C R$ function. It is crucial for the purpose of eventually reaching a consensus that the $F C R$ imposes a total order on all possible blockchains, such that conflicts are uniquely settled. Also note that $F C R$ is computed locally by all participants, with no communication needed. Intuitively, these two properties put together imply that if two participants have the same blocks in their local "block forests", they will agree wrt. what the correct global chain is. Extended to an entire network, this means that when all blocks have been delivered, all nodes decide upon the same chain. Or, in other words, all nodes are in consensus.

In the following sections, we establish this formally. 


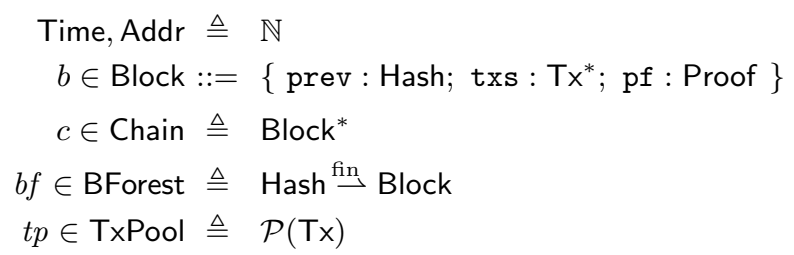

(a) Blocks, chains, and block forests

$$
\begin{aligned}
& \text { Hash, Proof, Tx : eqType } \\
& G B \text { : Block } \\
& \text { hash }_{b}: \text { Block } \rightarrow \text { Hash } \\
& \text { hash }_{t}: \mathrm{Tx} \rightarrow \text { Hash } \\
& \text { mkProof : Addr } \rightarrow \text { Chain } \rightarrow \text { option Proof } \\
& V A F: \text { Proof } \rightarrow \text { Time } \rightarrow \text { Chain } \rightarrow \text { bool } \\
& F C R: \text { Chain } \rightarrow \text { Chain } \rightarrow \text { bool } \\
& \text { tx Valid }: \mathrm{Tx} \rightarrow \text { Chain } \rightarrow \text { bool } \\
& \text { txExtend : TxPool } \rightarrow \mathrm{Tx} \rightarrow \text { TxPool }
\end{aligned}
$$

(b) Parameter primitive types and functions

Figure 2. Data structures and framework parameters.

\section{Data Structures for Blockchains}

We now present the data structures and primitives necessary for implementing the logic of the blockchain consensus protocol's replicated state machines.

\subsection{Parameters and Core Data Structures}

The top part of Figure 2 shows the definitions of the main data structures we are going to use. Some of the primitive data types are left undefined ( $c f$. Figure $2(\mathrm{~b})$ ), as they serve as parameters for the framework. For example, the types of time-stamps Time, necessary for modelling disciplines such as Proof-of-Stake, and network addresses Addr (ranged over by $\tau$ and $a$, correspondingly) are isomorphic to natural numbers $\mathbb{N}$. At the same time, the types Hash of hash values, proof objects Proof and transactions Tx (ranged over by $h, p f$, and $t x$, correspondingly) can be arbitrary (e.g., natural numbers, strings, etc) as long as they come with decidable equality checking, which is indicated by the eqType annotation.

Blocks are represented as records with three fields. The first one, prev, stores the hash of some block (even the very same one, although in most cases such blocks will be deemed ill-formed). The field txs stores a sequence of transactions, contained within this block, in the order they are supposed to take place. ${ }^{2}$ Finally, the proof object in pf is used to validate the block.

Two functions, hash $h_{t}$ and hash $_{b}$, for computing hashvalues of transactions and blocks correspondingly, are

\footnotetext{
${ }^{2}$ For simplicity, we consider transactions located in the same block to be non-conflicting with each other.
}

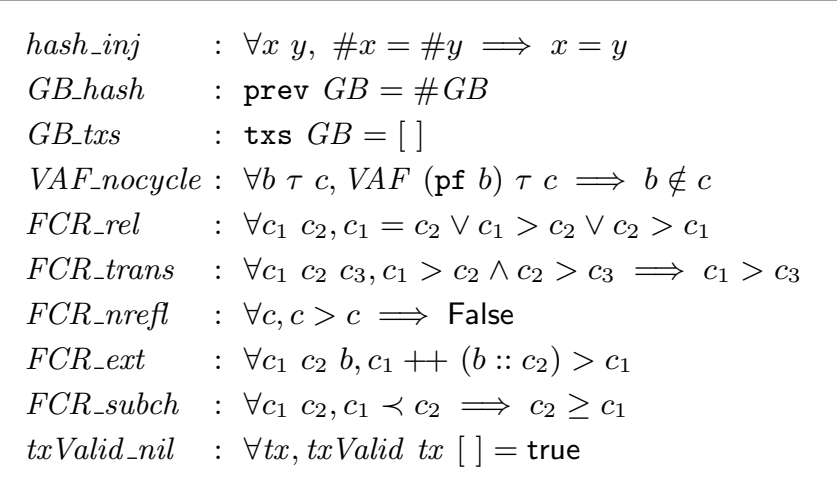

Figure 3. Axioms of the framework parameters.

provided by the client of the framework. For the sake of brevity, in the remainder of the paper, we will use the overloaded notation $\# x$ for computing the hash of a value $x$, which is either a block or a transaction, using the corresponding hash-function. The only requirement imposed on the hash function is it being injective, as stated by the axiom hash_inj in Figure 3.

We require the client of the framework to provide a dedicated Genesis Block GB, which is going to serve as an initial "seed" for all local ledgers, and is globally known in the system. This block is slightly different from other blocks we will observe in the system, as it is a subject of the first two hypotheses (axioms) we impose, which are shown in Figure 3. First, the hash of $G B$ should be equal to $G B$ 's prev value ( $G B \_h a s h$ ). This requirement seems quite artificial, but it is easy to achieve in practice by redefining the result of a typical hash-function for just one value, and it simplifies reasoning about forests, essentially ensuring that there can be no parent block of $G B$, in the presence of possible cycles between other (ill-formed) blocks referring to each other. An alternative to this construction would be to make a block's prev field optional, and ensure the GenesisBlock has no parent. The second axiom GB_txs ensures that the genesis block contains an empty transaction sequence [ ].

Blockchains (or simply chains) are defined as sequences of blocks, and block forests (or forests) are encoded as partial finite maps from hashes to blocks. The notions of "valid" chains and forests will follow below.

The next two parameter functions mkProof and $V A F$ work in tandem: the former is used to obtain a proof object for a specific node and on top of a particular chain, and it might fail, hence the option result type. In real-world blockchain implementations, computing a value of mkProof is an expensive operation, as it controls the number of valid blocks in the system, but here we do not model computational complexity, as it is irrelevant for establishing consensus, which is a safety, 
not a liveness property. Dually, $V A F$ is used to validate proof objects for a chain and it also takes a systemprovided time-stamp as an additional parameter. The only $V A F$-related axiom we need, $V A F \_$nocycle, ensures that a freshly "minted" (i.e., created) block $b$, for which a proof has been obtained wrt. to an "underlying" chain $c$, cannot be contained in the same $c$. An opposite situation would be an anomaly, and does not occur in real situations, in part due to the practical rareness of hash collisions.

The client-provided function $F C R$ allows one to compare the weights of two chains. From now on, we will abbreviate $\left(F C R c_{1} c_{2}\right)$ as $c_{1}>c_{2}$. The axioms $F C R_{-} r e l$, $F C R_{-}$trans, FCR_nrefl ensure that the order FCR imposes on chains is total, transitive, and irreflexive. The axiom $F C R_{-}$ext states that any non-empty extension of a chain $c_{1}$ produces a strictly "heavier" chain. In turn, $F C R_{-}$subch postulates that if a chain $c_{1}$ is a subchain of $c_{2}$ (i.e., $c_{1} \prec c_{2} \triangleq c_{2}=c^{\prime}++c_{1}++c^{\prime \prime}$ for some $c^{\prime}, c^{\prime \prime}$, such that at least one of $c^{\prime}$ and $c^{\prime \prime}$ is non-empty), then $c_{2}$ is at least as "heavy" as $c_{1}$.

Finally, the transaction validation function txValid ensures the absence of conflicts between a transaction $t x$ and a preceding chain $c$, being always true for an empty chain (as asserted by txValid_nil), and txExtend, which we did not have to constrain, is used to change a pool of pending transactions held by a particular node.

\subsection{Largest Chains and Block Forest Evolution}

Block forests are the main data structures nodes use to store incoming and locally minted blocks, and to reconstruct the actual ledger of transactions. The ledger of a forest $b f$, typeset as $\lceil b f\rceil$, is defined as the largest (wrt. $F C R$ ) chain starting at $G B$ and ending with some block $b$, which has a corresponding entry in $b f$.

How do we construct such a chain? To do so, we should restrict the class of forests bf we are working with to those satisfying the following three properties:

$$
\begin{aligned}
& \text { 1. } \forall h_{1}, h_{2} \in \operatorname{dom}(b f), h_{1}=h_{2} \Rightarrow b f\left(h_{1}\right)=b f\left(h_{2}\right) ; \\
& \text { 2. } \forall h b, b f(h)=b \Rightarrow h=\# b ; \\
& \text { 3. } b f(\# G B)=G B .
\end{aligned}
$$

The first property states that every key in $b f$ uniquely identifies its entry; the second ensures that for every block-entry in $b f$, its key is a hash of the corresponding block; finally, the third property makes sure that $b f$ contains the Genesis Block with its key. We define a forest $b f$ satisfying 1-3 as valid(bf) and will denote a block $b$ having a corresponding entry $\# b \mapsto b$ in a valid forest $b f$ as $b \in b f$, slightly abusing the $\in$-notation.

In the beginning of a system interaction, each node holds the same forest $b f_{0}=\{\# G B \mapsto G B\}$, which is trivially valid. As the nodes start minting new blocks and broadcast them, local forests might be extended with new blocks, for which we define the following operation:

$$
\begin{aligned}
& b f \triangleleft b \triangleq \\
& \quad \text { if } \# b \in \operatorname{dom}(b f) \text { then } b f \text { else } b f \uplus\{\# b \mapsto b\}
\end{aligned}
$$

That is, for any block, the result of $b f \triangleleft b$ is valid, if so was $b f$. Thus, fixing $\cdot \triangleleft \cdot$ as the only way to add a new block to a forest, in the rest of the paper we will be only dealing with valid forests, unless said otherwise.

Let us now compute the largest chain in a forest $b f$. Indeed, even a valid forest might not be a tree, due to gaps and possible cycles in the partial map that encodes it. We model cycles, even though they are implausible in a real-world setting, to account for the possibility that the hash functions used by the protocol might not be cryptographic. "Gaps", on the other hand, will appear frequently, as they correspond to blocks received out-oforder. To be considered a ledger candidate, a chain $c$ should satisfy the following conditions:

1. It should contain no duplicate blocks;

2. For any block $b \in c, b=G B$ or prev $b=b^{\prime}$, where $b^{\prime}$ is the block preceding $b$ in $c$;

3. The first block of $c$ should be $G B$ ( $g b$-founded $c)$;

4. For any block $b \in c$, and any transaction $t x \in \operatorname{txs} b$, txValid tx $c^{\prime}$ should be True, where $c^{\prime}$ is a prefix of $c$, preceding $b(t x$-valid $c)$.

To deliver such a candidate, we first construct a total function $\operatorname{chain}(b f, b)$ that returns a chain $c$, ending with a

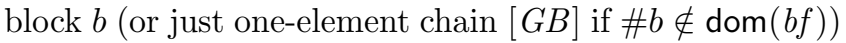
satisfying the conditions 1 and 2 above by implementing a "backwards walk" by prev-links from $b$ in $b f$ and ensuring that we do not visit the same block twice. Such a walk terminates if we encounter a cycle in $b f$ or we reach $G B$, which is its own previous block. The exact code of chain can be found in our supplementary Coq sources. Using chain, we construct candidates considering all blocks in bf and choose the largest one from those that satisfy conditions 3 and 4 . In set notation, for a valid $b f,\lceil b f\rceil$ is defined as follows:

$$
\lceil b f\rceil \triangleq \max _{F C R}\left\{\begin{array}{l|l}
c & \begin{array}{l}
c=\operatorname{chain}(b f, b), b \in b f, \\
g b \text {-founded } c \wedge \text { tx-valid } c
\end{array}
\end{array}\right\}
$$

The function $\lceil b f\rceil$ is implemented to be total so it returns $[G B]$ as a default ledger, if no better one is found.

To get a better intuition on the dynamics of $\lceil b f\rceil$ as the forest $b f$ keeps being extended with new blocks, let us take a look at Figure 4, which shows several states of a valid block forest with prev-links depicted by gray arrows. The stage (a) depicts a valid forest whose ledger is $c=[G B, A, B, C]$, with all other chains being less heavy or prefixes of $c$. In stage (b), due to out-of-order arrival, a block $G$ has been added to the forest, but at that moment it is orphaned, hence a chain built from it is not $g b$-founded. Once the missing block $F$ arrives in stage (c), a the forest gets a new ledger, namely $[G B, D, E, F, G]$, 


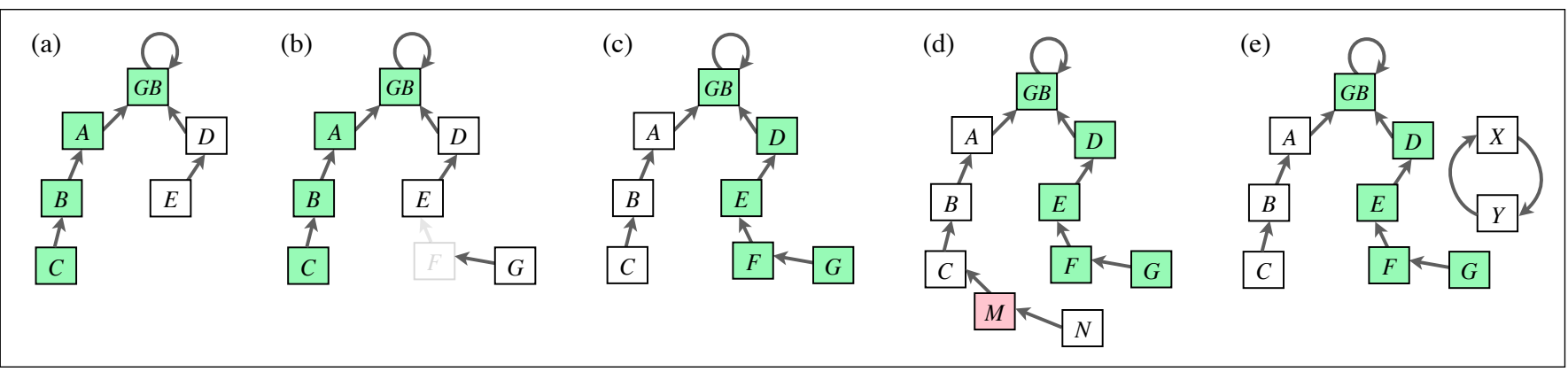

Figure 4. Different states of a valid block forest and its ledger chain (green) when extended with more blocks.

as it is more $F C R$-heavy than $[G B, A, B, C]$. Stages (d) and (e) show block forests that we account for in our implementation, but that will not correspond to local states of the protocol participants during a normal, nonByzantine execution (we discuss Byzantine cases in Section 7). Specifically, the forest in (d) has a block $M$, which is non-tx-valid wrt. [ $G B, A, B, C]$, and therefore does not contribute a ledger candidate, preventing all further chains in the forest that include it from being considered for the largest ledger. Finally, the case (e) demonstrates a cycle between two blocks, $X$ and $Y$, so neither of them is included into any chain to be considered a ledger candidate. In Section 4, we will show how the protocol prevents cases (d) and (e) from happening, when all participants are faithful.

\subsection{Local Forests and System-Wide Union}

The cases (a)-(c) from Figure 4 depict possible configurations of local forests, owned by particular participants of a blockchain protocol in the middle of a system's execution, when some of the blocks have already been received, while some others are in "in-flight" messages, i.e., yet to be delivered. Furthermore, in the absence of Byzantine participants, a system-wide union $\widehat{b f}$ of all locally owned forests will be defined, valid, and furthermore satisfy the following property, dubbed $\operatorname{good}(\widehat{b f})$ :

$$
\begin{aligned}
& \operatorname{good}(b f) \triangleq \forall b \in b f \\
& g b-f o u n d e d(\operatorname{chain}(b f, b)) \wedge t x-\operatorname{valid}(\operatorname{chain}(b f, b))
\end{aligned}
$$

In other words, a good forest is a tree, such that $G B$ is its root, and a chain built from any of its blocks starts with $G B$ and has no blocks with invalid transactions. A pleasant consequence of the fact $g b$-founded $(\operatorname{chain}(b f, b))$ is that the chain has no gaps, i.e., the result of chain $(b f, b)$ will not be affected by adding new blocks to $b f$, which is stated formally by the following lemma:

Lemma 3.1. For a valid forest $b f$ and a block $b$, if $g b$-founded $(\operatorname{chain}(b f, b))$ then for any block $b^{\prime}$, $\operatorname{chain}\left(b f \triangleleft b^{\prime}, b\right)=\operatorname{chain}(b f, b)$.
The following theorem is key for showing that locally minting a new block, when done right, always increases the local ledger, and, thus, has a chance to increase the global one, i.e., the ledger of a system-wide forest union.

Theorem 3.2. If a forest $b f$ is valid and a block $b$ is such that $t x$-valid $(\lceil b f\rceil, b)$, prev $b=\#($ last $\lceil b f\rceil)$, and $V A F(\mathrm{pf} b) \tau(\lceil b f\rceil)$ for some $\tau$, then $\lceil b f \triangleleft b\rceil>\lceil b f\rceil$.

That is, if a block $b$ is minted to extend the current $b f$ 's ledger, adding $b$ will deliver a heavier one.

For a (multi-)set of blocks $b s=\left\{b_{1}, b_{2}, \ldots, b_{n}\right\}$ and a forest $b f$, we define the operator $b f \ll b s$ as follows:

$$
\text { bf } \triangleleft\left\{b_{1}, b_{2}, \ldots, b_{n}\right\} \triangleq\left(\ldots\left(\left(b f \triangleleft b_{1}\right) \triangleleft b_{2}\right) \ldots\right) \triangleleft b_{n}
$$

The definition (2) of $\triangleleft$ implies that reordering or duplication of blocks in $b s$ does not affect the result of $\triangleleft \diamond b s$. We define the partial order $\sqsubseteq$ on valid forests as follows:

$$
b f_{1} \sqsubseteq b f_{2} \triangleq \exists b s, b f_{2}=b f_{1} \triangleleft b s
$$

We conclude this section with two theorems that are crucial for relating changes in a "node-local" ledger, due to minting a new block wrt. a node-local forest $b f$, and a "global" ledger built from the system-wide union of forests $\widehat{b f}$, such that $b f \sqsubseteq \widehat{b f}$. The first theorem states that, if a new block is global goodness-preserving, and if the new local ledger is heavier than the old global one, then the new local ledger is the new global one:

Theorem 3.3. For valid forests $b f, \widehat{b f}$ and a block $b$, if $\operatorname{good}(\widehat{b f})$, and $\operatorname{good}(\widehat{b f} \triangleleft b)$, and $\lceil b f \triangleleft b\rceil>\lceil\widehat{b f}\rceil$, and $b f \sqsubseteq \widehat{b f}$, then $\lceil b f \triangleleft b\rceil=\lceil\widehat{b f} \triangleleft b\rceil$.

The second theorem states that a correctly locally minted block $b$, if it does not contribute to create a ledger heavier than the current global one, will not change the global ledger even when added to the global forest:

Theorem 3.4. For valid forests $b f, \widehat{b f}$ and a block $b$, if $\operatorname{good}(\widehat{b f})$, and $\operatorname{good}(\widehat{b f} \triangleleft b)$, and tx-valid$(\lceil b f\rceil, b)$, and prev $b=\#($ last $\lceil b f\rceil)$, and $V A F$ (pf $b) \tau(\lceil b f\rceil)$, and $b f \sqsubseteq \widehat{b f}$, and $\lceil\widehat{b f}\rceil \geq\lceil b f \triangleleft b\rceil$, then $\lceil\widehat{b f} \triangleleft b\rceil=\lceil\widehat{b f}\rceil$. 
In a foresight of the development to be presented in the following sections, it should be intuitively clear that a ledger $\lceil\widehat{b f}\rceil$ of a system-wide forest union is what consensus is going to be reached upon. That is, each node, if it follows the rules of minting and adopting blocks, will eventually "align" its local ledger with $\lceil\widehat{b f}\rceil$.

\section{The Protocol and its Semantics}

From the description of the core data structures, we proceed to outline the logic of the protocol itself, represented as a family of replicated state-transition systems which communicate by asynchronously exchanging packets.

\subsection{System State-Space}

Figure 5 shows all state-space components of our system encoding. System configurations $\sigma$ are pairs of a global state $\Delta$ and a packet soup $P$. The former is a finite partial mapping from node addresses to their local states; described further below, while the latter is a (multi-)set of packets. ${ }^{3}$ Packets are simply triples, with the first component being the message sender, the second is the address of the destination, and the third one is the message content. We will further refer to the destination address of a package $p$ as dest $p$.

A local state $\delta$ of a node is a quadruple $\langle$ this, $a s, b f, t p\rangle$. Its first component this is the address of the node itself, which coincides with the key of this node in the system's global state; the second component is a set of addresses as representing the peers the node is aware of; the third component is a block forest $b f$, described in detail in Section 3, used to store the minted and received blocks; finally, the last component is a pool of transactions $t p$, storing locally created or received transactions to be included into minted blocks in the future.

Specific contents of the messages the nodes can send are defined by the data type Msg and include: NullMsg, which has no effect and is a message-passing analogue of the imperative skip command; ConnectMsg used by a node to announce itself to its peers; AddrMsg as used to propagate the set of peers as further into the system. TxMsg $t x$ is used to announce or propagate a new transaction $t x$, and BlockMsg $b$ serves the same purposes wrt. announcing a block $b$. InvMsg $h s$ is sent to inform others of the transactions and blocks a node holds locally (represented by their hashes $h s$ ); GetDataMsg $h$ is a request for a transaction or a block with a hash $h$, typically sent after having received an InvMsg.

In addition to emitting messages, nodes can perform internal operations, and we only capture the two that are relevant to the protocol in our semantics: creating

\footnotetext{
${ }^{3}$ Our semantics is resilient with respect to packet duplication, so here for simplicity we assume packet soups to be sets, while in or mechanisation they are modeled as multi-sets.
}

System configurations

$$
\begin{aligned}
\Delta \in \text { GlobState } & \triangleq \text { Addr } \stackrel{\text { fin }}{\rightarrow} \delta \\
P \in \text { PacketSoup } & \triangleq \mathcal{P}(\text { Packet }) \\
\sigma \in \text { Conf } & \triangleq \text { GlobState } \times \text { MessageSoup }
\end{aligned}
$$

\section{Local states}

$\delta \in$ LocState $\triangleq$ Addr $\times \mathcal{P}($ Addr $) \times$ BForest $\times$ TxPool

Messages, instructions and schedules

$$
\begin{aligned}
& p \in \text { Packet } \triangleq \text { Addr } \times \text { Addr } \times \text { Msg } \\
& m \in \text { Msg }::=\text { NullMsg } \\
& \mid \text { ConnectMsg } \\
& \mid \text { AddrMsg }(a s \in \mathcal{P}(\text { Addr })) \\
& \mid \text { TxMsg }(t x: \text { Tx }) \\
& \mid \text { BlockMsg }(b: \text { Block }) \\
& \mid \text { InvMsg }(h s \in \mathcal{P}(\text { Hash })) \\
& \mid \text { GetDataMsg }(h: \text { Hash }) \\
& i \in \text { Instr }:: \text { DoTx }(t x: \text { Tx }) \\
& \mid \text { DoMint } \\
& s \in \text { Selector }::=\text { Selldl } \\
& \mid \text { SelRcv }(a: \text { Addr }) \\
& \mid \text { Sellnt }(a: \text { Addr })(\tau: \text { Time })(i: \text { Instr }) \\
& s c \in \text { Schedule } \triangleq \text { Selector* }
\end{aligned}
$$

Figure 5. System state-space and schedules.

and announcing a transaction, and minting a new block. A data type Instr for instructions serves to encode these two operations. We will use instructions to encode nodespecific internal choices for modeling non-determinism in global network executions (described in Section 4.3) by parameterising them with schedules - finite sequences of selectors determining which node should act next and what is going to be its move.

\subsection{Local Node Semantics}

Figures 6 and 7 show the per-node transitions. While in our implementation they are encoded as executable Coq functions, in this paper we follow a more traditional relational style of presenting an operational semantics.

We split the semantics into receive-transitions and internal transitions. The former ones are of the form $\delta \stackrel{p}{\longrightarrow} \rho\left(\delta^{\prime}, p s\right)$ taking a node from a state $\delta$ and to $\delta^{\prime}$ when processing a package $p$, also emitting a new set of packages $p s$. The latter ones, $\delta \stackrel{\langle i, \tau\rangle}{\longrightarrow}\left(\delta^{\prime}, p s\right)$, describe change of a state from $\delta$ and $\delta^{\prime}$ with emission of packages $p s$ as a result of executing the instruction $i$ at a globally synchronised time $\tau$. 


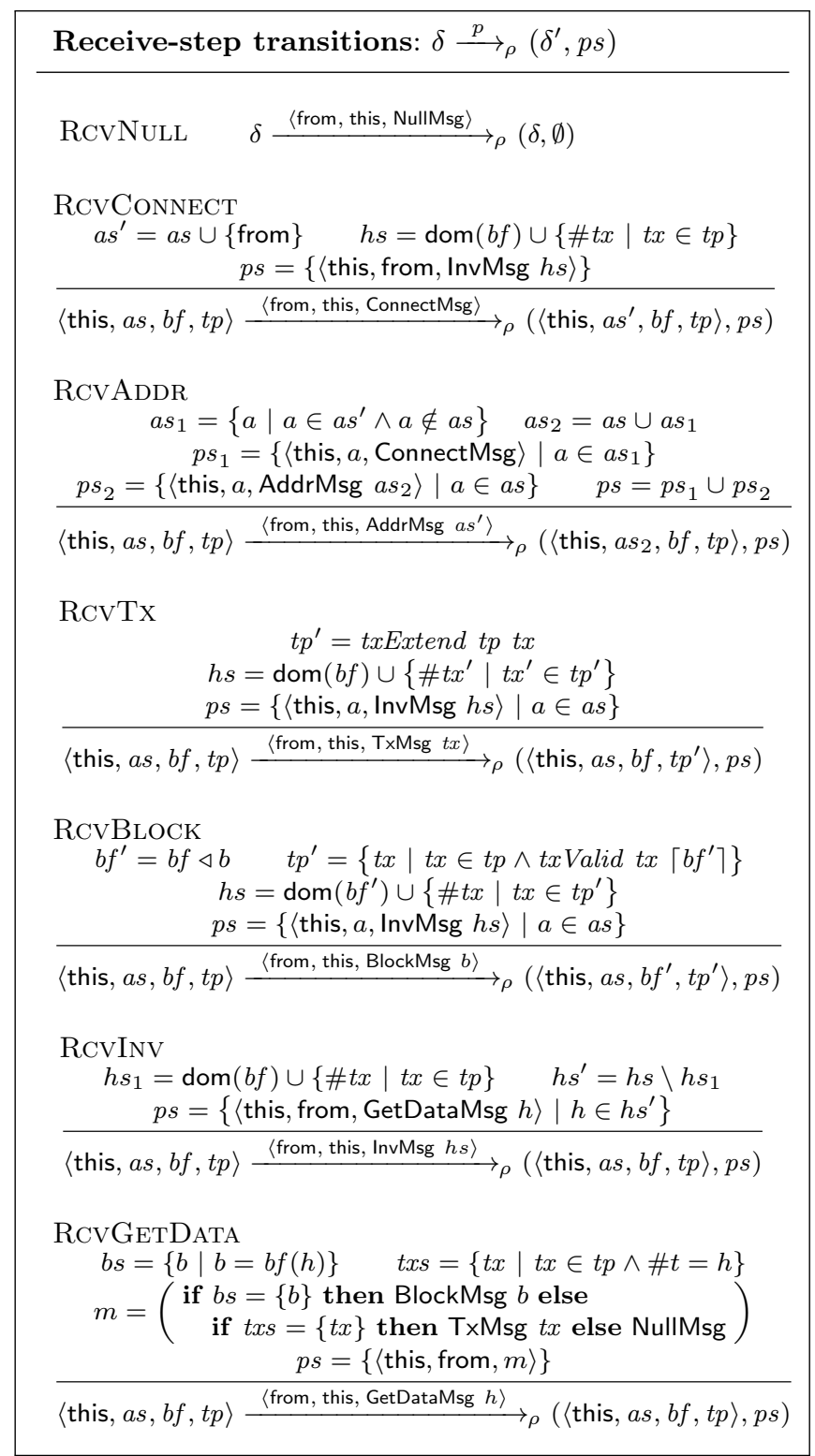

Figure 6. Local semantics, Part I: receive-transitions.

The receive-transitions (Figure 6) follow the intuition of the corresponding messages, and are mostly straightforward, so we only describe a few in prose. When taking a RCVADDR step, a node not only adds the new addresses to its local pool, but also sends a ConnectMsgrequest to the new peers it has learned about $\left(p s_{1}\right)$ and propagates the new information to its current peers $\left(p s_{2}\right)$, which will then themselves connect to the new peers (if they hadn't already). When receiving a new transaction or a block (via RCVTx or RCVBLOCK), a node adds it to its local state and informs its peers of now possessing it by sending an InvMsg, potentially causing a lot of redundant messages, which are nevertheless handled without

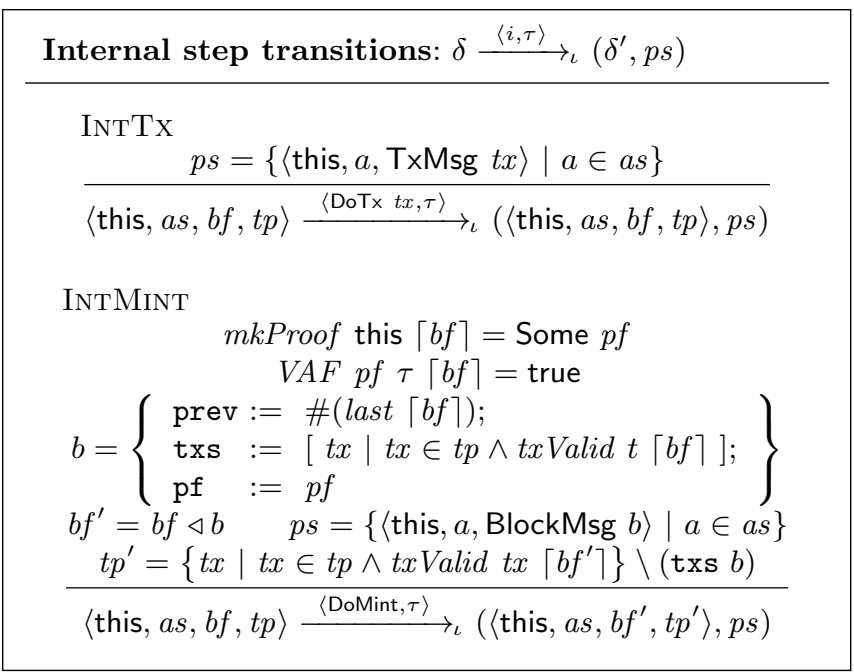

Figure 7. Local semantics, Part II: internal transitions.

any concerns for safety. Note that RCVBLOCK does not check whether the block it receives is valid before adding it to the local block forest. This seems unusual, but in reality is the only possible option, because a block's validity depends on the blocks that precede it, which the node may not yet have. Finally, the last two transitions serve to inform a node of new transactions and blocks in the system (via RCvINv), so it could request them by sending a GetDataMsg message, and the response to it will be sent (via RCvGetData) in the form of TxMsg or BlockMsg.

It is perhaps slightly non-obvious, but the rules allow to model the possibility of a node "joining late" and eventually "catching up" with the rest of the system, thanks to RCVCONNECT and other transitions that send known information about blocks transactions to the package origin from, so it could request them via RCvINV.

Figure 7 shows the two internal transitions that are triggered by the corresponding instructions. The INTTX simply adds a new transaction to the local pool, so it could be included into a block later, and announces it to the node's peers. The INTMinT transition relies on the block forest machinery and related primitives described in the previous section. Specifically, we (rather optimistically) assume that a node locally checks the new minted block $b$ with respect to its prefix chain, before adding it to its local forest and sending it to its peers.

With the rules in Figures 6 and 7, we intentionally define a non-optimal version of the protocol, such that nodes executing the transitions populate the packet soup with a lot of redundant messages. Yet, as we will show in Section 5, this does not pose problems for establishing consensus on the state of the global ledger. 
Network transitions: $\langle\Delta, P\rangle \stackrel{s}{\Longrightarrow}\left\langle\Delta^{\prime}, P^{\prime}\right\rangle$

$$
\begin{aligned}
& \text { NetDeliver } \\
& p \in P \quad \text { dest } p=a \quad \Delta(a)=\delta \quad \delta \stackrel{p}{\longrightarrow} \rho\left(\delta^{\prime}, p s\right) \\
& \langle\Delta, P\rangle \stackrel{\text { SelRcv } a}{\Longrightarrow}\left\langle\Delta\left[a \mapsto \delta^{\prime}\right], P \backslash\{p\} \cup p s\right\rangle \\
& \Delta(a)=\delta \quad \delta \stackrel{\langle i, \tau\rangle}{\longrightarrow}\left(\delta^{\prime}, p s\right) \\
& \langle\Delta, P\rangle \stackrel{\text { Sellnt } a \tau i}{=}\left\langle\Delta\left[a \mapsto \delta^{\prime}\right], P \cup p s\right\rangle
\end{aligned}
$$

$$
\text { NEtIDLE }\langle\Delta, P\rangle \stackrel{\text { Selldl }}{\Longrightarrow}\langle\Delta, P\rangle
$$

Figure 8. Network semantics.

\subsection{Network Semantics}

The network semantics rules, parameterised by a selector $s$, are shown in Figure 8. They are standard for modeling interleaved concurrency with non-deterministic internal choices and message delivery. The three rules account for a possibility of delivering a randomly picked package $p$ from the soup $P$ to a destination $a$ (NetDeliver), a node $a$ taking an internal step with an instruction $i$ (NetInternal) or doing nothing (NetIdLe).

While the rules do not change the global set of node addresses, we nevertheless can model a scenario of a node "joining" the network, assuming that it already has a predefined address and a correctly initialised initial state, so it only needs to announce itself to its peers and requests the information about transactions and blocks. ${ }^{4}$

We conclude this section by defining the notion of reachability $(\rightsquigarrow)$ between two configurations as follows:

$$
\begin{aligned}
\sigma \rightsquigarrow \sigma^{\prime} \triangleq & \sigma=\sigma^{\prime} \vee \\
\exists s c= & {\left[s_{1}, \ldots, s_{n}\right],\left[\sigma_{1}, \ldots, \sigma_{n-1}\right], \text { s.t. } } \\
& \quad \stackrel{s_{1}}{\Longrightarrow} \sigma_{1} \wedge \ldots \wedge \sigma_{n-1} \stackrel{s_{n}}{\Longrightarrow} \sigma^{\prime} .
\end{aligned}
$$

\section{System Safety and Consensus}

With the definitions of the protocol and a library of theorems about block forests at hand, we are now ready to establish several important safety properties, including the eventual consistency (i.e., the consensus) of our system. It is customary to formulate safety properties as inductive system invariants, defined as follows:

Definition 5.1. The property $I$ : Conf $\rightarrow$ Prop is an inductive invariant of a system if for the system's initial configuration $\sigma_{0}, I\left(\sigma_{0}\right)$ holds, and for any $\sigma, \sigma^{\prime}$ and $s$, such that $I(\sigma)$ holds, $\sigma \stackrel{s}{\Longrightarrow} \sigma^{\prime}$ implies $I\left(\sigma^{\prime}\right)$.

\footnotetext{
${ }^{4}$ We could have added another internal transition rule for emitting a ConnectMsg, but this is orthogonal to our study of system safety.
}

Therefore, by induction, an inductive property $I$ will hold for any system configuration $\sigma$, such that $\sigma_{0} \rightsquigarrow$ $\sigma$. Indeed, what can be proven inductive depends on the choice of the initial system state, which we have not specified so far. For the rest of this section, we will consider only the initial configurations of the form $\sigma_{0}=\left\langle\right.$ GlobState $\left._{0}, \emptyset\right\rangle$, where for any $a \in \operatorname{dom}\left(\right.$ GlobState $\left._{0}\right)$, GlobState $_{0}(a)=\left\langle a, a s_{a},\{\# G B \mapsto G B\},\{\}\right\rangle$, i.e., leaving only the node-specific sets of peers $a s_{a}$ unconstrained.

\subsection{System State Coherence}

Before moving to the interesting (and, hence, complex) system safety properties, we start by establishing the inductivity of global state coherence, i.e., proving that interaction between nodes does not violate the validity of the components of each node's local state. We thus define the global system state coherence as follows:

$$
\begin{aligned}
\operatorname{Coh}(\langle\Delta,-\rangle) \triangleq \forall a \in \operatorname{dom}(\Delta), \exists \text { as bf tp, } \\
\Delta(a)=\langle a, \text { as, bf, tp }\rangle \wedge \operatorname{valid}(b f)
\end{aligned}
$$

The validity of each local forest $b f$ is via the definition (1). Any of the $\sigma_{0}$ we consider satisfies it, and the property Coh is inductive, because all manipulations with nodelocal block forests are done using the $\triangleleft$ operation (2).

\subsection{Eventual Ledger Consistency}

Let us now formulate the eventual consistency of the system. Informally, it says that when there are no inflight messages between any of the nodes, they all should agree on the local ledger, which can be, thus, thought of as a globally shared one [32].

In practice, however, communication between nodes never stops. Our protocol features many "modes of communication" (announcing a block, requesting hashes, etc), and, as it turns out, not all of them should be ceased for reaching consensus on ledgers. What is essential is to have no in-flight instances of BlockMsg. 5 Having no in-flight block-messages, however, is not the only requirement for the universality of the consensus (i.e., ensuring that each two nodes have the same ledger): it might be the case that some nodes joined late, and due to the delays in updating the topology, have not yet requested all missing data from their peers. Characterising consistency conditions in this case would require us to take the "late joiners" into account. While not impossible, this would make the whole consistency statement quite complicated. To avoid this, in this paper we decided to formulate the consistency in a simpler setting: a clique network topology, restricting the initial configurations to those where every node's known peers include all addresses in the global state. ${ }^{6}$

\footnotetext{
${ }^{5}$ The version we present is a form of quiescent consistency $[1,4]$. ${ }^{6}$ This situation is quite common for corporate blockchain-based protocols, where all peers know each other from the very beginning.
} 


$$
\begin{aligned}
& \operatorname{Cliq}(\langle\Delta, P\rangle) \triangleq \\
& \operatorname{Coh}(\langle\Delta, P\rangle) \wedge \\
& \forall a \in \operatorname{dom}(\Delta), \operatorname{dom}(\Delta) \subseteq \operatorname{peers}(\Delta, a) \wedge \\
& \exists c, \widehat{b f}, \widehat{a} \in \operatorname{dom}(\Delta) \text {, such that } \\
& \text { (i) } \forall a \in \operatorname{dom}(\Delta), \widehat{b f}=\operatorname{forest}(\Delta, a) \triangleleft \operatorname{blocksFor}(P, a) \wedge \\
& \text { (ii) } \operatorname{valid}(\widehat{b f}) \wedge \operatorname{good}(\widehat{b f}) \wedge c=\lceil\widehat{b f}\rceil \\
& \text { (iii) } \forall a \in \operatorname{dom}(\Delta), c \geq \operatorname{ledger}(\Delta, a) \\
& \text { (iv) } \operatorname{ledger}(\Delta, \widehat{a})=c \\
& \text { where } \operatorname{peers}(\Delta, a) \triangleq a s \text {, s.t. }\langle a, a s,-,-\rangle=\Delta(a) \\
& \operatorname{forest}(\Delta, a) \triangleq b f \text {, s.t. }\langle a, b f,-,-\rangle=\Delta(a)
\end{aligned}
$$

Figure 9. Cliq system property.

We embed the clique topology assumption into the whole-system property Cliq, whose formal definition we postpone until Section 5.3. For now, let us present the eventual consistency result it implies. For this, we introduce two auxiliary definitions. The first one extracts a ledger for a node $a \in \operatorname{dom}(\Delta)$ from a global state $\Delta$.

$$
\text { ledger }(\Delta, a) \triangleq\lceil b f\rceil \text {, s.t. }\langle a,-, b f,-\rangle=\Delta(a)
$$

The second returns all in-flight blocks for $a$ in a soup $P$ :

$$
\text { blocksFor }(P, a) \triangleq\{b \mid\langle-, a, \text { BlockMsg } b\rangle \in P\}
$$

The desired theorem is as follows:

Theorem 5.1 (Consensus in a clique topology). For $\sigma=\langle\Delta, P\rangle$, if Cliq $(\sigma)$ holds, then there exists a chain $c$, such that for any node $a \in \operatorname{dom}(\sigma), c \geq \operatorname{ledger}(\Delta, a)$ and $\operatorname{blocksFor}(P, a)=\emptyset$ implies ledger $(\Delta, a)=c$.

The chain $c$ from Theorem 5.1 statement is a globally shared ledger, and in a quiescent state, all nodes have it.

\subsection{Clique Invariant}

We now show the statement of the Cliq property, highlight its key insights, and convey the intuition of the proof that it is indeed inductive for systems that start in initial configurations $\sigma_{0}$ with a clique network topology.

The formal definition of Cliq, with the most important conjuncts labelled $(i)-(i v)$ is given in Figure 9. The first two non-labelled conjuncts ensure that the property holds over configurations that are coherent (8) and have a clique topology, as discussed above. The rest of the definition is more interesting, as it exhibits an important property of blockchain-based protocols, which we call the law of block conservation. The "conservation" is expressed via the existence of a global forest $\widehat{b f}$ (foreshadowed as a system-wide forest union in Section 3.3), which is a superset of the local forest of any node $a$, as stated by conjunct $(i)$, and can be obtained by adding all blocks currently in-flight towards $a$ to $a$ 's local forest. The global forest $\widehat{b f}$ is also valid (1), good (4) and has the "canonical" ledger $c(i i)$, which is larger or equal than any local ledger (iii). Finally, there is always a node $\widehat{a} \in \operatorname{dom}(\Delta)$ that has the canonical ledger $c$, even though $\widehat{a}$ 's local forest might be a strict subset of $\widehat{b f}(i v)$. The statement of Theorem 5.1 trivially follows from (i) $-(i i)$ as then the subject node's forest is exactly $\widehat{b f}$.

Why is Cliq inductive? In our Coq development, we have proved that Cliq is preserved by the network semantics. The proof is of interest, as it heavily relies on the idempotence of the $\triangleleft$ operation, and the "goodness" of the global forest $\widehat{b f}$, whose ledger $c$ (owned by at least one node in the network) serves as the constructive witness of what the consensus is going to be reached upon. The trickiest parts of the proof concern "restoring" conjuncts $(i i)$ and $(i v)$ when an arbitrary node takes the INTMinT transition, with a chance of either (a) becoming the new owner $\widehat{a}$ of the global ledger $c$, or (b) minting a block that is already in $\widehat{b f}$ or simply does not deliver a heavier chain. The case (a) is handled by Theorem 3.3, while the case (b) is what is delivered by Theorem 3.4. The following theorem therefore holds:

Theorem 5.2. For systems that initially have a clique network topology, Cliq is an inductive invariant.

On the clique assumption. What would the invariant and the eventual consistency statements look like without the clique assumption? At the moment, the definition of Cliq ensures that for any node $a$ and any block $b$ in the system, $b$ is either already in $a$ 's local forest or is "flying towards" it. With this assumption, our proofs do not rely on the more advanced features of the protocol, such as peer-exchange (via AddrMsg and ConnectMsg) and on-demand data exchange (via InvMsg and GetDataMsg). These features will become useful in the future, when we want to prove more interesting invariants. To illustrate this, let us consider a case of a node $a^{\prime}$ that has joined late, announcing itself (via ConnectMsg) only to a few other participants. Then, $a^{\prime}$ might not have yet requested or has not yet been forwarded all the blocks already minted in the system. Therefore, in order to relate its local state to $\widehat{b f}$, we would have to enhance the invariant with a conjunct for ongoing "propagation" of the known peers in the system, and replace $(i)$ by it. In addition to that, we would need to consider situations when the topology is not a connected graph, in which case several "canonical" chains would co-exist without ever being reconciled. Stating the consensus property in such settings is our future work.

\section{Elements of our Mechanisation}

We mechanised all results described in this paper in Coq, making use of the Ssreflect/MathComp libraries [22, 30]. Our implementation of block forests builds on the 
Table 1. Sizes of definitions and proofs (LOC).

\begin{tabular}{|c||c|c|}
\hline & Definitions & Proofs \\
\hline Block Forests & 579 & 1406 \\
Protocol and Network & 409 & 263 \\
Consensus Properties & 241 & 273 \\
\hline
\end{tabular}

library of partial finite maps by Nanevski et al. [24]. The size of our contributed codebase is pleasantly small, as demonstrated by the lines of code figures in Table 1.

In the proofs, we heavily relied on the small-scale reflection and rewriting machinery provided by Ssreflect [11]. For instance, our implementation of block forests features both constructive and computable definitions of prefix/fork relations on chains, as well as the corresponding reflect-view lemmas, to switch between the two representations. The definitions of all operations and predicates on block forests, such as (1)-(3), and on network configurations, such as (9) and (10), are also made decidable/computable. This design choice has paid off not only in reducing proof sizes, but also in the robustness of our proof scripts in the face of changes made in the definitions, which is surprising given how modest the amount of automation we used in the project was.

As an anecdote from our experience, while preparing this submission, a few days before the CPP'18 deadline and already after having completed proofs of all invariants, we have noticed an odd encoding of the rule RCVConneCT. In our mechanisation to date, the corresponding transition was only adding the sender from to the local list of peers, but, rather selfishly, did not send a list of available hashes back as an InvMsg. We have changed the implementation so it would precisely match the rule from Figure 6, and, to our surprise, no proofs of invariants broke. We consider this an encouraging sign to invest more time into domain-specific automation for proofs about replicated state-transition systems.

\section{Discussion}

We now discuss the limitations of our protocol model and the implications of the assumptions we made.

Network semantics and system faults. As defined in Section 4, our network semantics is quite restricted. For instance, it does not include notions of packets being dropped or of participant faults. In practice, the clique assumption means that we can largely ignore crash faults, as we do not need other participants to relay our messages and we do not expect to receive any responses.

A possible complication arises when a participant crashes while in the process of broadcasting a newlyminted block, such that some peers receive it and others do not. This scenario, which is very similar to that of dropped packets, is difficult to accommodate in the current invariant. However, the problem with dropped packets would essentially disappear once we start making use of the protocol's peer-to-peer facilities, as they a provide a large amount of communication redundancy. That is, participants in the network advertise their entire knowledge every time they update it, and they request information they do not have from all peers that have advertised it. For reaching the consensus eventually, it is sufficient that one of these messages gets delivered. If none of them is, the process repeats the next time a peer updates its state and advertises.

Byzantine behaviours. A special case of faults is that of Byzantine faults, in which participants exhibit arbitrary behaviour [20]. These may arise due to software bugs, hardware malfunctions or through the actions of malicious actors. Our invariant is not resistant to Byzantine faults. For example, the proof relies on the fact that all blocks in the canonical block forest are tx-valid. This is true under normal operation, but can be invalidated at will by a malicious actor. In order to reason about the ineffectiveness of Byzantine faults, we will have to introduce to the invariant some notion of honest participants being in the majority and in communication with each other, and to find a way of accommodating within the proofs the presence of "bad" blocks.

It is important to stress that these invalid blocks, as seen in cases (d) and (e) of Figure 4, do not in any way prevent the protocol from operating correctly (from the perspective of faithful participants), but merely make it more difficult to prove that it does.

Other protocol properties. In this work, we have focused exclusively on the safety of the system, i.e., the property that all correct nodes agree wrt. which ledger they adopt. Other properties, such as liveness and various security properties, depend on the choice of system parameters hash, $V A F$, and FCR. For example, system security almost certainly requires that we use a cryptographic hash function, i.e., a hash function that is both collision-resistant (approx. injective) and pre-image resistant (given $h(m)$, finding $m$ is computationally hard). For liveness, we will at the very least need to ensure, in the form of a new axiom, that hashes for blocks do not collide with hashes for transactions. Otherwise, information might not propagate correctly throughout the network. Moreover, we likely want $V A F$ to impose a reasonable delay between consecutive block mintings, such that messages have time to propagate throughout the network - this would provide an adequate quiescent state, and thus consensus can be reached. Studying the full implications of different framework parameters is left for future work. 
Towards a verified blockchain implementation. Our implementation of the protocol is intentionally nonoptimal. Whenever faced with a decision of how to implement a function, we always chose simplicity over efficiency. That being said, all of our functions of, e.g., processing block forests, are pure, so that they can be replaced with more efficient functional or imperative equivalents. For example, the reference implementation of chain performs a lot of redundant computation and would greatly benefit from a memoization strategy. Similarly, the message propagation strategy is very inefficient, and could possibly be replaced with a more sensible one.

Because our mechanisation of the protocol is encoded as a library of computable Coq functions that implement state transformers and message handlers, it should be possible to extract it to OCaml and run on top of a trusted shim implementation, thus providing a formally verified blockchain implementation, in the same way it has been done in the recent work on the DISEL framework [31, 34]. This setup, however, appears quite naïve and would be problematic in a realistic case of Byzantine faults, as the safety results established in this work hold only as long as all participants follow the protocol and use exactly the same version of the shim, which is hard to guarantee in an adversarial distributed environment.

\section{Related Work}

The results we presented in this paper are related to the latest advances in the areas of computer security, formal methods, and distributed systems.

\subsection{Consistency of Blockchain Protocols}

In the past few years, there has been a lot of interest within the security and privacy community for notions of consistency in application to blockchain protocols.

Garay et al. considered the core protocol underlying Bitcoin [23], focusing on its two properties, dubbed Common Prefix and Chain Quality [10]. The common prefix property is a probabilistic version of the notion of eventual consistency we have established in Section 5 of this paper. Specifically, they establish that all honest parties in the system agree on a common ledger prefix up to $k$ last blocks, where $k$ is a parameter of the system. The chain quality property tackles a Byzantine setting in which malicious participants may contribute ill-formed blocks, and states that the number of such blocks in the system in not very large, given that the majority of participants remain honest and follow the protocol. Unlike our formalization, the work by Garay et al. takes into the account possible adversarial behaviours of the protocol participants, but restricts the communication to fully synchronous, i.e., messages in the system are instantly delivered without delays, whereas we allow for arbitrary delays and permutations in message delivery.

While that work focuses on proving the properties of Nakamoto's consensus based on Proof-of-Work [23], in a follow-up to that result, Kiayias et al. propose a blockchain consensus protocol based on Proof-of-Stake [2] and possessing the same properties, and also a new one, Chain Growth, which ensures overall liveness for the honest parties [19], under the assumption of synchronous message delivery in the network. Finally, in a recent work, Pass et al. provided probabilistic boundaries with respect to chain growth and quality, as well as the analysis of other consistency and liveness properties of blockchain consensus in a fully asynchronous environment [26].

In contrast with those and many other works $[6,10,19$, 26 ] that analyse blockchain consensus from the perspective of security properties, thus, focusing on probabilistic reasoning about a protocol modeled as a composition of distributions, we present a simple operational model that immediately provides an executable semantics of the system, but only allows us to prove "coarse-grained" correctness conditions, such as eventual consistency.

None of the proofs of security properties of blockchain consensus we are aware of were mechanised.

\subsection{Formal Methods for Blockchains}

To date, the interest of the formal methods community wrt. blockchain-based systems is predominantly in applications of the technology, rather than reasoning about properties of the underlying protocols.

In the past two years, a number of works have been published on formal modeling and verification of smart contracts - a mechanism to associate executable code with certain blockchain transactions, providing a machinery for trusted decentralised arbitration, which gained a lot of attention thanks to its highly influential implementation in Ethereum [36]. Various formal semantics of Ethereum Virtual Machine (EVM) and its contract language Solidity were implemented in Coq [17], Isabelle [16], $\mathrm{F}^{\star}$ [3], Idris [27], $\mathrm{K}$ [14], Why3 [9], and in custom tools for static and dynamic analysis [21].

An implementation of an efficient data structure for transaction ledgers has been developed and verified in Coq by White [33], yet it has not been used in the context of verifying a protocol that employs this structure.

At the level of reasoning about protocols, Hirai has formalised a simple variant of a Proof-of-Stake protocol in Isabelle [15], proving a version of the protocols' $a c$ countable safety: if two conflicting blocks get adopted in a shared block tree, then at least $\frac{1}{3}$ of participants may lose their entire deposits (stakes). This property is specific to Ethereum's Casper protocol [5] and is orthogonal to the consensus result we established in this work. 


\subsection{Verification of Distributed Consensus}

Several recent major efforts have fully mechanised and verified implementations of more traditional consensus protocols, such as versions of Paxos [7, 13, 18, 25, 29], Raft [35, 37], or the classical Two-Phase Commit [31, 34]. Even though none of those works consider blockchain consensus, we believe, many of those frameworks can handle it, as long as they can adopt our model and support reasoning about block forests. Therefore, we see our main conceptual contribution in distilling the protocol semantics and outlining the proof layout for blockchain consensus.

\section{Conclusion and Future Work}

In this work, we have presented a formal operational model of a distributed blockchain-based consensus protocol, implemented its core data structures, characterised the primitives it relies upon, and mechanically proved a form of the protocol's eventual consistency, i.e., that a system that implements it does indeed reach a consensus.

In the future, we are going to enhance our mechanisation for reasoning about relevant security properties [19], modeling schedule-providing oracles as probabilistic distributions. We also plan to define an operational semantics for transactions run on top of the protocol, using it as a foundational platform for verified smart contracts.

\section{Acknowledgments}

We thank the CPP'18 reviewers for the careful reading and constructive suggestions on the paper and the formalisation. We also thank June Andronick and Amy Felty for their efforts as CPP'18 Program Co-Chairs.

Sergey's research was supported by EPSRC Grant EP/P009271/1 "Program Logics for Compositional Specification and Verification of Distributed Systems".

\section{References}

[1] James Aspnes, Maurice Herlihy, and Nir Shavit. 1994. Counting Networks. J. ACM 41, 5 (1994), 1020-1048.

[2] Iddo Bentov, Ariel Gabizon, and Alex Mizrahi. 2014. Cryptocurrencies without Proof of Work. CoRR abs/1406.5694 (2014).

[3] Karthikeyan Bhargavan, Antoine Delignat-Lavaud, Cédric Fournet, Anitha Gollamudi, Georges Gonthier, Nadim Kobeissi, Natalia Kulatova, Aseem Rastogi, Thomas SibutPinote, Nikhil Swamy, and Santiago Zanella-Béguelin. 2016. Formal Verification of Smart Contracts: Short Paper. In PLAS ACM, 91-96.

[4] Sebastian Burckhardt, Alexey Gotsman, Hongseok Yang, and Marek Zawirski. 2014. Replicated data types: specification, verification, optimality. In POPL. ACM, 271-284.

[5] Vitalik Buterin and Virgil Griffith. 2017. Casper the Friendly Finality Gadget. CoRR abs/1710.09437 (2017).

[6] Phil Daian, Rafael Pass, and Elaine Shi. 2017. Snow White: Robustly reconfigurable consensus and applications to provably secure proofs of stake. Technical Report. Cryptology ePrint Archive, Report 2016/919.

[7] Cezara Dragoi, Thomas A. Henzinger, and Damien Zufferey. 2016. PSync: a partially synchronous language for faulttolerant distributed algorithms. In POPL. ACM, 400-415.

[8] Cynthia Dwork and Moni Naor. 1992. Pricing via Processing or Combatting Junk Mail. In CRYPTO (LNCS), Vol. 740. Springer, 139-147.

[9] Jean-Christophe Filliâtre and Andrei Paskevich. 2013. Why3 - Where Programs Meet Provers. In ESOP (LNCS), Vol. 7792. Springer, 125-128.

[10] Juan A. Garay, Aggelos Kiayias, and Nikos Leonardos. 2015. The Bitcoin Backbone Protocol: Analysis and Applications. In EUROCRYPT (Part 2) (LNCS), Vol. 9057. Springer, 281310 .

[11] Georges Gonthier, Assia Mahboubi, and Enrico Tassi. 2009. A Small Scale Reflection Extension for the Coq system. Technical Report 6455. Microsoft Research - Inria Joint Centre.

[12] L.M. Goodman. 2014. Tezos: A Self-Amending CryptoLedger. Position Paper. https://www.tezos.com/static/papers/ position_paper.pdf. (2014).

[13] Chris Hawblitzel, Jon Howell, Manos Kapritsos, Jacob R. Lorch, Bryan Parno, Michael L. Roberts, Srinath T. V. Setty, and Brian Zill. 2015. IronFleet: proving practical distributed systems correct. In SOSP. ACM, 1-17.

[14] Everett Hildenbrandt, Manasvi Saxena, Xiaoran Zhu, Nishant Rodrigues, Philip Daian, Dwight Guth, and Grigore Rosu. 2017. KEVM: A Complete Semantics of the Ethereum Virtual Machine. Technical Report.

[15] Yoichi Hirai. 2017. A mechanized safety proof for PoS with dynamic validators. https://medium.com/@pirapira/ a-mechanized-safety-proof-for-pos-with-dynamic-validators-17e9b45faff4. (2017).

[16] Yoichi Hirai. 2017. Defining the Ethereum Virtual Machine for Interactive Theorem Provers. In 1st Workshop on Trusted Smart Contracts.

[17] Yoichi Hirai. 2017. Ethereum Virtual Machine for Coq (v0.0.2). https://medium.com/@pirapira/ ethereum-virtual-machine-for-coq-v0-0-2-d2568e068b18. (2017).

[18] Mauro Jaskelioff and Stephan Merz. 2005. Proving the Correctness of Disk Paxos. Archive of Formal Proofs 2005 (2005).

[19] Aggelos Kiayias, Alexander Russell, Bernardo David, and Roman Oliynykov. 2017. Ouroboros: A Provably Secure Proofof-Stake Blockchain Protocol. In CRYPTO (Part 1) (LNCS), Vol. 10401. Springer, 357-388.

[20] Leslie Lamport, Robert E. Shostak, and Marshall C. Pease. 1982. The Byzantine Generals Problem. ACM Trans. Program. Lang. Syst. 4, 3 (1982), 382-401.

[21] Loi Luu, Duc-Hiep Chu, Hrishi Olickel, Prateek Saxena, and Aquinas Hobor. 2016. Making Smart Contracts Smarter. In CCS. ACM, 254-269.

[22] Assia Mahboubi and Enrico Tassi. 2017. Mathematical Components. Available at https://math-comp.github.io/mcb.

[23] Satoshi Nakamoto. 2008. Bitcoin: A peer-to-peer electronic cash system. http://bitcoin.org/bitcoin.pdf. (2008).

[24] Aleksandar Nanevski, Viktor Vafeiadis, and Josh Berdine. 2010. Structuring the verification of heap-manipulating programs. In POPL. ACM, 261-274.

[25] Oded Padon, Giuliano Losa, Mooly Sagiv, and Sharon Shoham. 2017. Paxos made EPR: decidable reasoning about distributed protocols. PACMPL 1, OOPSLA (2017), 108:1-108:31. 
[26] Rafael Pass, Lior Seeman, and Abhi Shelat. 2017. Analysis of the Blockchain Protocol in Asynchronous Networks. In EUROCRYPT (Part 2) (LNCS), Vol. 10211. Springer, 643673.

[27] Jack Pettersson and Robert Edström. 2016. Safer Smart Contracts through Type-Driven Development. Master's thesis. Chalmers University of Technology, Department of Computer Science and Engineering, Sweden.

[28] George Pîrlea and Ilya Sergey. 2017. Toychain: a Coq implementation of a minimalistic blockchain-based consensus protocol. A version accepted at CPP 2018 is available at https://github.com/certichain/toychain/tree/cpp2018. (2017).

[29] Vincent Rahli, David Guaspari, Mark Bickford, and Robert L. Constable. 2015. Formal Specification, Verification, and Implementation of Fault-Tolerant Systems using EventML. In AVOCS. EASST.

[30] Ilya Sergey. 2014. Programs and Proofs: Mechanizing Mathematics with Dependent Types. Lecture notes with exercises. Available at http://ilyasergey.net/pnp.

[31] Ilya Sergey, James R. Wilcox, and Zachary Tatlock. 2018. Programming and Proving with Distributed Protocols. PACMPL 2, POPL (2018), 28:1-28:30.
[32] Douglas B. Terry, Marvin Theimer, Karin Petersen, Alan J. Demers, Mike Spreitzer, and Carl Hauser. 1995. Managing Update Conflicts in Bayou, a Weakly Connected Replicated Storage System. In SOSP. ACM, 172-183.

[33] Bill White. 2015. A Theory for Lightweight Cryptocurrency Ledgers. (8 February 2015). Unpublished draft. Code available at https://github.com/input-output-hk/qeditas-ledgertheory, last accessed on 10 October 2017.

[34] James R. Wilcox, Ilya Sergey, and Zachary Tatlock. 2017. Programming Language Abstractions for Modularly Verified Distributed Systems. In SNAPL (LIPICs), Vol. 71. Schloss Dagstuhl - Leibniz-Zentrum fuer Informatik, 19:1-19:12.

[35] James R. Wilcox, Doug Woos, Pavel Panchekha, Zachary Tatlock, Xi Wang, Michael D. Ernst, and Thomas E. Anderson. 2015. Verdi: a framework for implementing and formally verifying distributed systems. In PLDI. ACM, 357-368.

[36] Gavin Wood. 2014. Ethereum: A secure decentralised generalised transaction ledger. http://gavwood.com/paper.pdf. (2014).

[37] Doug Woos, James R. Wilcox, Steve Anton, Zachary Tatlock, Michael D. Ernst, and Thomas E. Anderson. 2016. Planning for change in a formal verification of the Raft Consensus Protocol. In CPP. ACM, 154-165. 